\title{
Second-order wave maker theory using force-feedback control. Part I. A new theory for regular wave generation.
}

\author{
Johannes Spinneken and Chris Swan \\ Imperial College London, Department of Civil and Environmental Engineering \\ London SW7 2AZ
}

2009

\begin{abstract}
Second-order wave maker theory has long been established; the most extensive and detailed approach given by Schäffer (1996). However, all existing theories assume the wave paddle is driven by a positionfeedback motion controller. Early research in the wave power field led to the design of a force-controlled absorbing wave machine (Salter 1982). In addition to operating as an excellent absorber, this machine seemed to introduce very little spurious harmonic content when driven with a first-order command signal. The present paper provides a mathematical model for the operation of wave makers using force-feedback control and seeks to explain this apparent advantage. The model is developed to second-order so that a command signal compensating for the remaining spurious wave is also provided. Due to the complexity of the problem, the model has been limited to flap-type wave machines and the generation of regular waves. A variety of numerical tests in force-control mode have been conducted, indicating that the spurious wave content is greatly reduced when compared to the position-control mode. A separate experimental study validating the theory is presented in a part II paper by the same authors.
\end{abstract}

Keywords: Laboratory wave generation, Second-order wave maker theory, Force-feedback control, Impedance matching.

\section{Introduction}

Position-feedback has traditionally been used for controlling the behaviour of wave machines. Indeed, the dominance of this approach is such that the term 'first-order control signal' has almost become synonymous with 'first-order position control signal'. In the context of force-controlled wave machines, the understanding of the control quantity needs to be broadened. Throughout this paper 'position-controlled' refers to machines where the wave board position is the only control parameter, while 'force-controlled' refers to machines where the force acting on the wave board is one of the controlled quantities; the others being position, velocity and acceleration.

The behaviour of position-controlled wave machines is well understood. A first-order theory was initially derived by Havelock (1929). Second-order effects such as long waves in laboratory models have been discussed by Ottesen-Hansen et al. (1980), Sand (1982) and Sand \& Donslund (1985). In addition, Flick \& Guza (1980) and Sulisz \& Hudspeth (1993) addressed the effects of spurious superharmonic waves. In seeking an explanation of these effects, Schäffer (1996) derived a complete mathematical model for position-controlled wave makers including the sub- and superharmonic effects arising at second-order.

With advances in laboratory wave generation techniques, wave makers are increasingly required to provide active absorption in order to avoid spurious reflection and reduce the flume or basin stilling time. Reviews on this extensive topic are given by Schäffer \& Klopman (2000) and Naito (2006). Historically, Milgram (1970) pioneered this field for both theory and practical application. Although his final experimental apparatus used a signal based on the surface elevation measured some distance from the absorbing terminal, he also suggested using the wave-induced force acting on the face of the wave board to implement active absorption. However, due to concerns regarding the resonant excitation of the fluid behind the wave paddle, he decided 
it would be impractical to use the force signal.

Research in the wave power field (Salter et al. 1976) led to a detailed investigation of the forces acting on various oscillating bodies. Because this work was often carried out by electrical engineers, the concept of an impedance was commonly adopted. In essence, the impedance describes a frequency depended function between the incident force and the oscillator velocity; the latter being the mechanical analogies to voltage and current. Impedance in the context of hydrodynamics has been discussed in McCormick et al. (1981) and McCormick (1983). Falnes (1980) showed that for the special case of a single oscillator system, the optimum oscillation velocity for maximum absorption is in phase with the excitation force. This condition can be achieved using an impedance matching controller; further discussion of this being given in $\S 2$. To overcome the problematic resonant fluid motion behind the paddle, described by Milgram (1970), Salter (1982) engineered a dry-backed paddle so that the measured force solely depends on the wave-induced pressure acting on the paddle face and the dynamics of the wave board.

In addition to operating as excellent absorbers, the dry-backed force-controlled wave machines have a significant benefit over position-controlled machines in that they seem to introduce very little spurious harmonic content. As a result, in excess of 500 force-controlled wave paddles are operating today in many laboratory facilities worldwide. Even though these devices have been used for more than three decades, a complete theory, linking the demand signal to the generated wave field, has not been derived. In practice, the machines are set up using an empirical tank transfer function. The successful application of this method, as well as an improved calibration technique, has been reported by Masterton \& Swan (2008).

The main objective of the present paper is to derive a mathematical model predicting the behaviour of forcecontrolled wave machines up to second order. At first order, this model seeks to link the linear demand signal to the linear or first-order wave field; hence, at the very least, providing an initial estimate for the empirical transfer function mentioned above. At second order, the model aims to explain the machines success in terms of the absence of spurious wave modes.

The proposed model will build upon existing wave maker theories: the wave field generated by a given sinusoidal paddle motion is identical for both position- and force-controlled machines. However, a single harmonic force-control signal does not inevitably lead to a paddle motion at this harmonic alone. Indeed, all the nonlinear processes in the generated wave field will contribute to the applied force and hence feed back into the generation process. A full explanation of this effect is given in $\S 2$. Once the behaviour up to second order is fully understood, an additional command signal for the suppression of the remaining spurious superharmonic may be evaluated.

Unlike for position-controlled machines, absorption is integral to the operation of force-controlled machines. As such, the second-order model derived here, includes all effects introduced by the absorption mechanism. Schäffer \& Jakobsen (2003) (see also Zhang (2005)) derived a new theory of non-linear wave generation in position-control with active absorption. However, for large reflections, they state that the theory is not fully consistent with regard to non-linearity. The full impact of absorption filters on non-linear wave generation in position-control has not been explained yet. Consequently, the force-feedback model developed herein is compared to non-absorbing position-controlled machines.

In a variety of numerical tests in force-control mode $(\S 3)$ it is shown that the spurious wave content introduced in the wave flume is greatly reduced when compared to the position-control mode. A set of experiments substantiating the numerical tests is presented in Spinneken \& Swan (2008b), hereafter referred to as part II.

\section{Theory for force-controlled absorbing wave makers}

\subsection{Physical Model}

For position-controlled machines, the control quantity is easily defined: the mechanics of the paddle and, more importantly, the generated wave field do not affect the board motion. In contrast, with a force-controlled wave machine the control quantity is not so straightforward. Even though the force is a crucial part of the control, the position, velocity and acceleration of the wave machine are also important. In order to fully understand this concept, the wave machine has to be considered as a coupled mass-spring-damper system; the forces appropriate to such a system being dependent on position, velocity and acceleration. In addition to the forces related to the generated wave field, hereafter referred to as the hydrodynamic feedback, the paddle spring, damping and inertia also have to be modelled precisely. 
To understand the complexity of the hydrodynamic feedback, it is important to appreciate the following. Applying a single-frequency, sinusoidal force to the paddle will cause it to move and generate a wave field. This wave field, in turn, induces a force on the wave board which is sensed by a force transducer. The nature of this force is dependent on the full nonlinearity of the waves and it is this nonlinear force that determines, via the paddle controller, the force to be applied in the next time step. Consequently, exciting a force-controlled wave machine at one frequency, will cause a nonlinear or multiple frequency paddle motion. This is in marked contrast to a position-controlled wave machine.

Furthermore, absorption is achieved using an impedance-matching digital filter network. These filters compute the wave board velocity signal or the first derivative of the board position. In accordance with the discussion above, the velocity signal may contain harmonics of the primary generation signal which will contribute to the total feedback signal. With these two simultaneous feedback paths (force and filtered velocity), force-controlled wave machines cannot be modelled considering only one process quantity at a time; hence the earlier comment that absorption is an integral part of a force-controlled system. As a result, a complete frequency domain model, including hydrodynamic feedback, wave board dynamics as well as absorption filters, must be derived. Each of these aspects are considered in the following subsections.

\subsection{Hydrodynamic feedback}

Assuming the first-order motion of the paddle is known, the generated wave field up to second order can be derived. This has been discussed extensively (Flick \& Guza (1980), Sulisz \& Hudspeth (1993), van Leeuwen \& Klopman (1996), Schäffer (1996) and Zaman \& Mak (2007)) and will not be repeated herein. Indeed, throughout this paper the notation and solution as presented by Schäffer (1996) will be adopted. With the wave field known, the wave-induced force acting on the paddle can be derived, representing part of the input to the paddle controller.

\subsubsection{Generated wave field}

The potential solution for a wave field generated by a second-order force signal can be separated into four components:

i. The first-order velocity potential - $\Phi^{(1)}$

This describes the progressive wave to be generated as well as the associated evanescent modes arising at, or near, the wave paddle. It must satisfy the boundary condition on the wave maker, requiring the fluid velocity normal to the wave board to be equal to the wave board velocity. The velocity potential can be expressed in terms of the wave board motion following Schäffer (1996). Relating the first-order force input to the first-order wave board motion is one of the key objectives of the work presented herein.

ii. The wave-wave interaction potential - $\Phi^{(21)}$

In the general case of an irregular sea state, the various first-order waves and their evanescent modes described by $\Phi^{(1)}$ will interact with each other; $\Phi^{(21)}$ describes these cross interaction terms as well as the self interaction or Stokes terms. With the present study limited to regular waves, only the self- and evanescent interaction terms are important.

iii. The spurious free wave potential $-\Phi^{(22)}$

Although $\Phi^{(1)}$ and $\Phi^{(21)}$ together satisfy the second-order free surface boundary conditions, they do not fully satisfy the second-order boundary condition at the wave maker. This leads to the generation of an unwanted free wave which is expressed as $\Phi^{(22)}$. For the special case of a regular wave, the spurious content at some distance from the paddle, where the evanescent modes have decayed, is a pure superharmonic of the primary wave.

iv. Feedback involving a second-order second harmonic correction - $\Phi^{(23)}$

The sum of all the forces at second harmonic is measured by the machine's force transducer. Even if there is no second harmonic specified in the command signal, the feedback excites the paddle at this frequency. The resulting paddle motion generates another freely propagating spurious wave. In order to generate the desired wave only $\left(\Phi^{(1)}+\Phi^{(21)}\right)$, the contributions $\Phi^{(22)}$ and $\Phi^{(23)}$ must cancel each other out. The additional second-harmonic command force must be evaluated to satisfy this condition. 


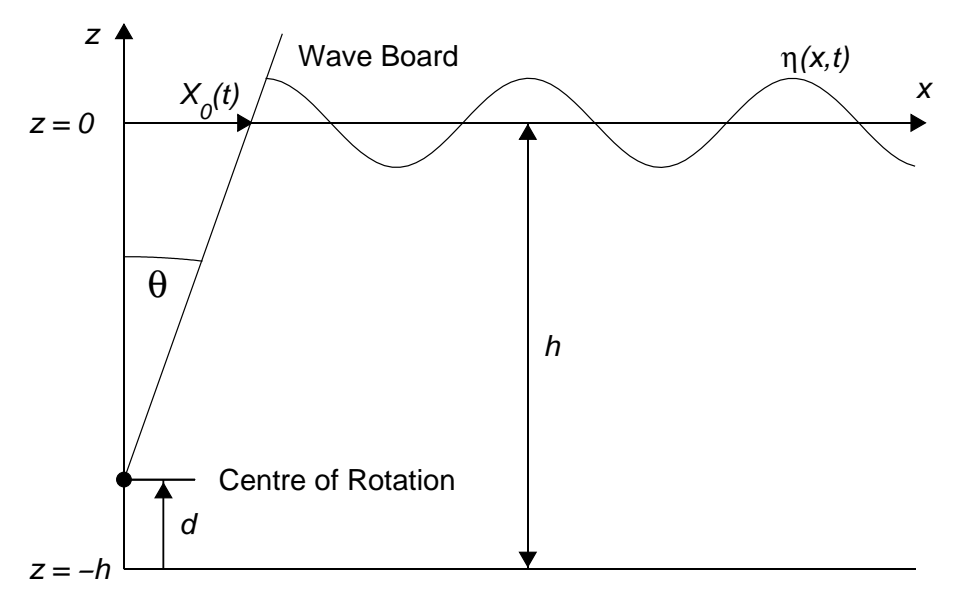

Figure 1: Wave maker geometry

The solution of the first three potentials is identical to that presented by Schäffer (1996). In contrast, $\Phi^{(23)}$ results from the wave maker moving at the second harmonic caused by the nonlinear feedback and the additional correction force. In order to determine the complex amplitude of the feedback-induced motion, the entire system has to be modelled.

\subsubsection{First-order solution}

Schäffer (1996) derived a boundary-value problem, based on a classical perturbation approach combined with Taylor expansions of the boundary conditions at the free surface. Adopting similar notation: the velocity potential $\Phi=\Phi(x, z, t)$ in Cartesian coordinates $(x, z)$ is defined such that the velocity components are given by $(u, w)=\left(\Phi_{x}, \Phi_{z}\right)$, the water surface elevation is given by $\eta=\eta(x, t)$ and the wave board position by $X=X(z, t)$, with $\omega, g, h$ and $t$ denoting angular wave frequency, acceleration of gravity, still water depth, and time, respectively. The length $l$ is defined so that $z=-(h+l)$ gives the centre of rotation of the wave board and $d$ is the elevation of the hinge above the bed. For the flap-type wave machines discussed herein it is unnecessary to introduce both $d$ and $l$, because $l=-d$ for all cases. However, for consistency with Schäffer and to enable future expansion of the theory this notation will be adopted. Figure 1 defines both the wave maker geometry and the notation employed.

The boundary conditions appropriate to the problem defined on figure 1 are as follows:

a. With the bed assumed to be horizontal and impermeable, $w=0$ on $z=-h$.

b. The water surface profile is a streamline defining the Kinematic Free Surface Boundary Condition (KFSBC).

c. The pressure acting on the water surface is constant (atmospheric) defining the Dynamic Free Surface Boundary Condition (DFSBC)

d. On the wave board, the fluid velocity perpendicular to the wave board must be equal to the velocity of the wave board

Evaluating these boundary conditions for an inviscid and incompressible fluid, yields the first-order wave potential given by

$$
\Phi^{(1)}=\frac{1}{2}\left\{\frac{i g X_{a}}{\omega} \sum_{j=0}^{\infty} c_{j} \frac{\cosh k_{j}(z+h)}{\cosh k_{j} h} e^{i\left(\omega t-k_{j} x\right)}+c . c .\right\},
$$



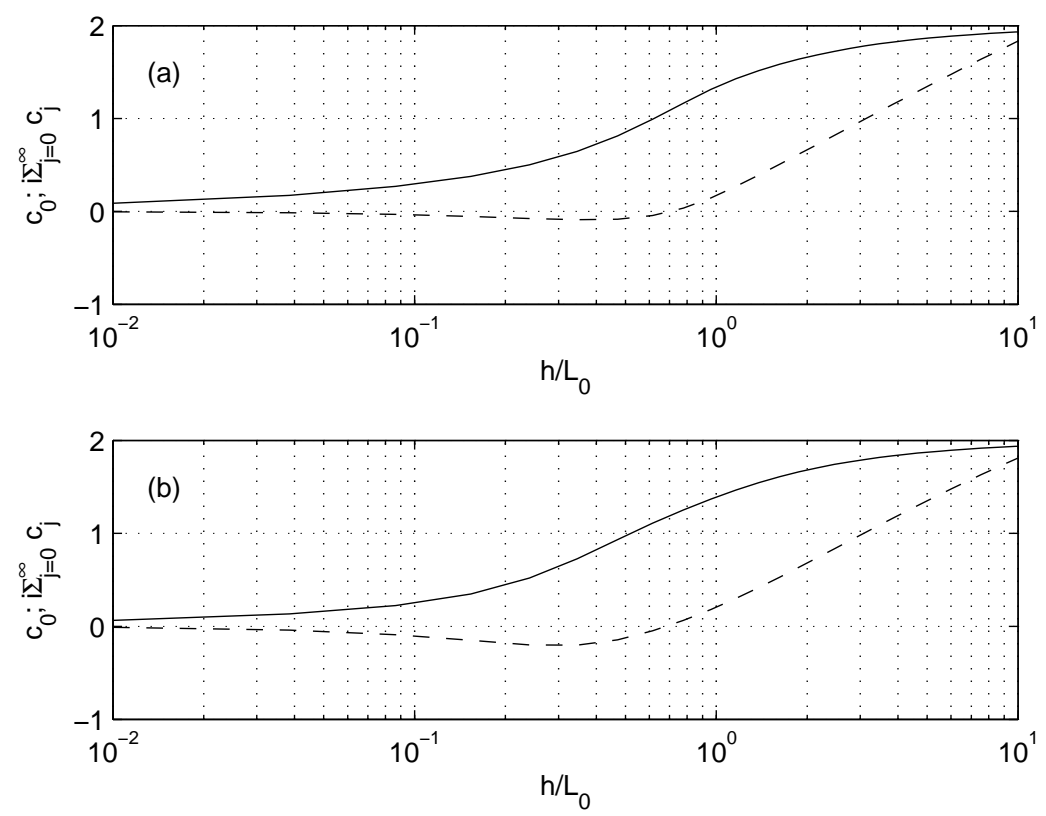

Figure 2: Dimensionless first-order coefficients; $(a)$ wave maker hinged at $d / h=0 ;(b)$ wave maker hinged at $d / h=0.5 ;-c_{0}$, characterising the propagating wave mode; $--i \sum_{j=0}^{\infty} c_{j}$, representing the sum of the evanescent modes

where $X_{a}$ is the complex, first-order, paddle displacement amplitude at still water level and c.c. the complex conjugate of the preceding term. Using this result, the first-order paddle motion $X_{0}^{(1)}$ may be defined as

$$
X_{0}^{(1)}=\frac{1}{2}\left\{-i X_{a} e^{i \omega t}+c . c .\right\} .
$$

The wave numbers, $k_{j}$, are the solutions to the dispersion equation

$$
\omega^{2}=g k_{j} \tanh k_{j} h,
$$

generalized to complex wave numbers (Dean \& Dalrymple 1991). Within this solution, $j=0$ refers to the progressive part of the wave field whilst $j=1 \ldots \infty$ refers to the infinite series of evanescent modes; the latter accounting for the mismatch between the wave board velocity and water particle velocity under the progressive wave. This involves satisfying the boundary condition on the wave board (condition $(d)$ above) and yields the geometry dependent first-order coefficients $c_{j}$; an analytic expression of $c_{j}$ is provided in Appendix A. This paper will address two types of machines: bottom hinged wave makers $(l=0, d=0)$ and wave makers where the hinge is placed above the bed $(l=-d<0)$. Figure 2 shows the first-order coefficients for these two different arrangements, where $L_{0}=2 \pi g / \omega^{2}$ is the deep water wave length.

\subsubsection{Second-order solution}

Figure 3 shows the dimensionless ratio between the spurious free wave amplitude $\left(A^{(22)}\right.$ arising from $\left.\Phi^{(22)}\right)$ and the Stokes second-order wave amplitude $\left(A^{(21)}\right.$ arising from $\left.\Phi^{(21)}\right)$ at some distance from the wave paddle for the two flap wave machines discussed in figure 2. The curves are based on the second-order solution given by Schäffer (1996). Simplifying his results for the special case of regular waves yields

$$
\Phi^{(21)}=\frac{1}{2}\left\{\frac{i X_{a}^{2}}{2} \sum_{j=0}^{\infty} \sum_{l=0}^{\infty} \frac{H_{j l}^{+}}{D_{j l}^{+}} c_{j} c_{l} \frac{\cosh \left(k_{j}+k_{l}\right)(z+h)}{\cosh \left(k_{j}+k_{l}\right) h} e^{i\left(2 \omega t-k_{j} x-k_{l} x\right)}+c . c .\right\}
$$




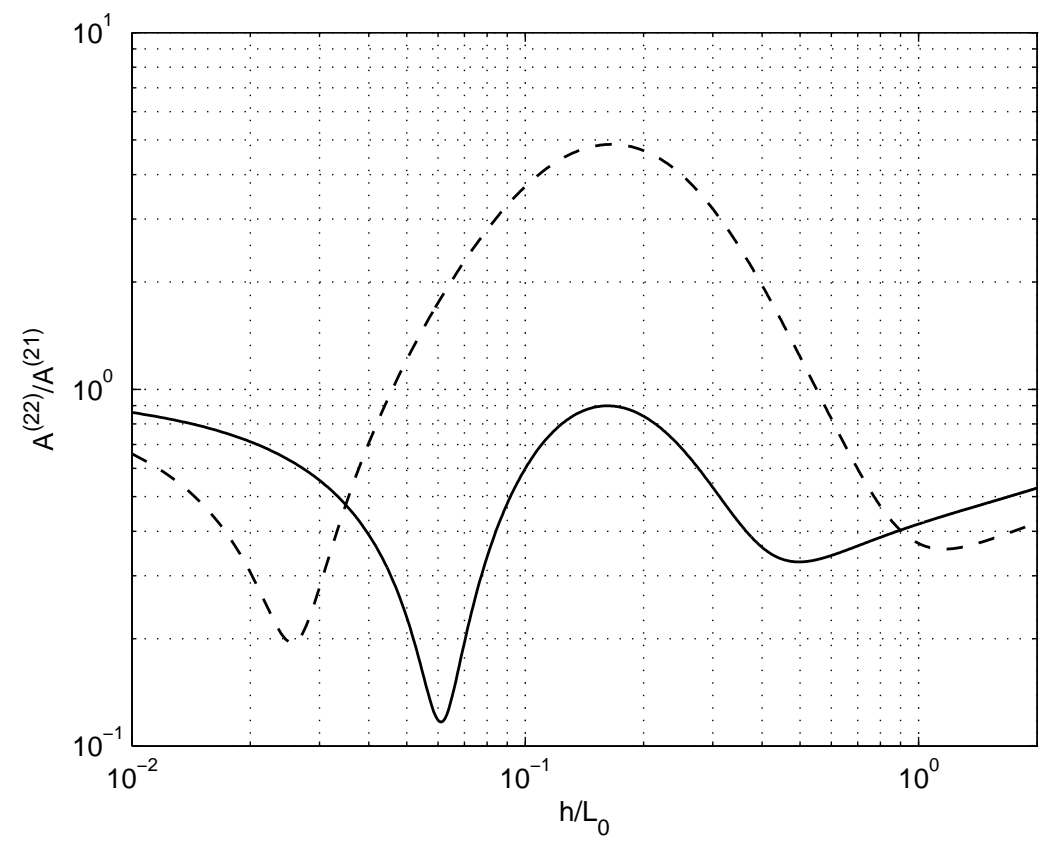

Figure 3: Dimensionless ratio between spurious free wave amplitude, $A^{(22)}$, and Stokes second-order wave amplitude, $A^{(21)} ;-$ wave maker hinged at $d / h=0 ;--$ wave maker hinged at $d / h=0.5$

and

$$
\Phi^{(22)}=\frac{1}{2}\left\{\frac{i g c_{0}^{2} X_{a}^{2}}{2 h \omega} \sum_{p=0}^{\infty} c_{p}^{(22)+} \frac{\cosh K_{p}^{+}(z+h)}{\cosh K_{p}^{+} h} e^{i\left(2 \omega t-K_{p}^{+} x\right)}+c . c .\right\},
$$

with $H_{j l}^{+}, D_{j l}^{+}, K_{p}^{+}$and $c_{p}^{(22)+}$ given in Appendix A; the corresponding surface elevations, $\eta^{(21)}$ and $\eta^{(22)}$, being given by Schäffer (1996). The curves shown in figure 3 are also in close agreement with the theory derived by Sulisz \& Hudspeth (1993).

In the case of a bottom hinged wave machine $(d / h=0)$ the spurious wave may be as large as the Stokes second-order content and peaks at $h / L_{0} \approx 0.15$. This is a relatively shallow water wave and the Stokes contribution is hence significant, leading to a sizable spurious free wave. In contrast, the minimum occurs at $h / L_{0} \approx 0.06$. However, the latter point is only of theoretical interest as it would be inappropriate to operate a flap machine in this regime; the small first-order coefficient $c_{0}$ in figure 2 (a) indicates that a very large stroke would be required to generate a wave of practical amplitude. In this case, a piston-type wave maker would be required.

Similar arguments apply in the second wave maker case $(d / h=0.5)$. The minimum $A^{(22)} / A^{(21)}$ occurs in very shallow water $h / L_{0} \approx 0.03$; whilst the maximum again occurs at $h / L_{0} \approx 0.15$. In this latter case, a spurious free wave of up to four times the Stokes content arises. This is clearly undesirable because this region is of interest for wave generation (figure $2 \mathrm{~b}$ ).

As the water depth increases, the dimensionless spurious wave ratio becomes small for both paddle configurations. However, this may be of little practical importance, since the Stokes second-order effects are less pronounced in this regime unless extremely steep waves are generated.

\subsubsection{Hydrodynamic torques on flap-type wave machines}

In the case of a flap-type wave machine, the origins of the feedback potential $\Phi^{(23)}$ are best described by considering the applied torque, $T$. A wave-induced pressure acting at the hinge of the flap makes no contribution to the measured hydrodynamic torque, whilst a pressure acting at or above still water level has 
a very large effect. In order to evaluate the total hydrodynamic torque, Bernoulli's equation, given by

$$
p(z, t)=-\rho \frac{\partial \Phi}{\partial t}-\rho g z-\frac{1}{2} \rho\left[\left(\frac{\partial \Phi}{\partial x}\right)^{2}+\left(\frac{\partial \Phi}{\partial z}\right)^{2}\right]
$$

is applied throughout the wave field and integrated over depth, where $\rho$ is the density of the fluid and $p(z, t)$ is the depth dependent pressure fluctuation. Within the present study, interest lies in torques up to second order; terms of higher order being neglected. Separating the total torque into its first- and second-order components gives

$$
T_{\Phi}^{(1)}=-\int_{-h+d}^{0} \rho \frac{\partial \Phi^{(1)}}{\partial t}(h-d+z) d z
$$

and

$$
\begin{aligned}
T_{\Phi}^{(2)}=-\int_{0}^{\eta^{(1)}} \rho g z(h-d+z) d z-\int_{0}^{\eta^{(1)}} \rho \frac{\partial \Phi^{(1)}}{\partial t}(h-d+z) d z & \\
-\int_{-h+d}^{0} \frac{\rho}{2}\left(\frac{\partial \Phi^{(1)}}{\partial x}\right)^{2} & (h-d+z) d z-\int_{-h+d}^{0} \frac{\rho}{2}\left(\frac{\partial \Phi^{(1)}}{\partial z}\right)^{2}(h-d+z) d z \\
& \quad-\int_{-h+d}^{0} \rho \frac{\partial \Phi^{(21)}}{\partial t}(h-d+z) d z-\int_{-h+d}^{0} \rho \frac{\partial \Phi^{(22)}}{\partial t}(h-d+z) d z,
\end{aligned}
$$

where the subscript $\Phi$ indicates wave-induced quantities and the superscript () denotes the order of the term involved. All torques are derived as torques per unit paddle width. Further detail concerning the derivation of these results is given in Appendix B. In addition to the wave-induced contributions to the torque, flap-type machines also experience a torque caused by the varying effective water depth associated with departures from the mean or vertical position. The total hydrostatic torque is given by

$$
T_{h}=-\frac{1}{6} \rho g(h-d)^{3} \frac{1}{\cos ^{2} \theta} \approx-\frac{1}{6} \rho g(h-d)^{3}\left(1+\theta^{2}\right)
$$

which may be approximated as the sum of a static term and a second order contribution, $T_{h}^{(2)}$. With flaptype wave machines typically being operated in the range of \pm 0.3 radians, the second-order approximation introduces an error of less than one percent for the maximum stroke. The total second-order torque is now given by

$$
T^{(2)}=T_{\Phi}^{(2)}+T_{h}^{(2)}
$$

where $T^{(2)}$ is the forcing term leading to the generation of the additional second-order wave potential $\Phi^{(23)}$. However, the systems transfer function, incorporating the wave board dynamics and the absorption filter, needs to be known before the impact of $T^{(2)}$ can be evaluated.

\subsection{Wave board and wave dynamics}

The torques discussed above are all induced by the generated wave field. In practice, a transducer would sense three torque components: the hydrodynamic feedback torque, the wave board inertia torque and the wave board gravity torque. When modelling the behaviour of the wave machine, the paddle is regarded as an inverted pendulum with inertia, $r$, and a gravity induced component, $-m g h_{m}$, where $h_{m}$ is the distance from the hinge to the centre of gravity and $m$ is the paddle's mass. Considering a physical pendulum, the gravity induced component varies with $\sin \theta$. However, with flap-type wave machines typically being operated in the range of \pm 0.3 radians, the first-order approximation, $\sin \theta \approx \theta$, introduces an error of less than two percent over the full range, neglecting terms at third order and above. This gravity induced torque is crucial for very low frequencies, whereas the inertia torque may become dominant towards the higher frequencies. The equation of motion for this system is given by

$$
\left(r+r_{\Phi}\right) \ddot{\theta}+d_{\Phi} \dot{\theta}-m g h_{m} \theta=T,
$$

where the mechanical damping, $d$, is assumed to be negligible compared to the hydrodynamic damping, $d_{\Phi}$, and the coefficients for added inertia, $r_{\Phi}$, and hydrodynamic damping will be derived from the wave field solution in the next section. The total wave board induced torque $\left(r \ddot{\theta}-m g h_{m} \theta\right)$ is hereafter referred to as wave board dynamic torque. 


\subsection{Force based wave absorption}

Absorption is an integral part of force-feedback controlled wave machines; the method applied being based on an approach comparable to impedance matching in signal transmission. Falnes (1993) states two conditions for maximum power absorption: the condition for optimum phase and the condition for optimum amplitude. In the special case of a single oscillator system, in the present case a single flap wave maker with one degree of freedom, Falnes (1980) has shown that the optimum oscillation velocity for maximum absorption is in phase with the excitation force. Furthermore, maximum power is absorbed from the wave when the destructive interference between the incident wave and the radiated wave is largest. Combining the two conditions gives the expression for maximum power absorption

$$
Z_{f}=Z_{d}^{*}
$$

where the impedance $Z$ can be decomposed into $Z=R+i Y$, with $R$ being the radiation resistance and $Y$ the radiation reactance. Furthermore, $Z^{*}$ denotes the complex conjugate of $Z$, while the subscripts $f$ and $d$ denote filter and dynamic respectively. The result, given in (12) is well known from elementary theory for electrical circuits. The dynamic impedance, $Z_{d}$, includes both wave induced quantities as well the wave board dynamics. In practice, the filter impedance, $Z_{f}$, is adjusted using a network of electrical (digital) filters. As this does not provide an exact match to the complex conjugate dynamic impedance, $Z_{d}^{*}$, over the full frequency range, the resulting reflection coefficient has to be calculated. Following Falnes (2002) the absorbed power, $P_{a}$, is given by

$$
P_{a}=\frac{R_{f} / 2\left|T_{e}\right|^{2}}{\left(R_{f}+R_{d}\right)^{2}+\left(Y_{f}+Y_{d}\right)^{2}}
$$

where $T_{e}$ is the excitation torque. Applying the optimum phase and amplitude conditions, (13) evaluates to

$$
P_{a, \max }=\frac{\left|T_{e}\right|^{2}}{8 R_{d}}
$$

for the maximum absorbed power. Introducing the power reflection coefficient, $\gamma_{r}$, and substituting (13) and (14) yields

$$
\gamma_{r}=1-\frac{P_{a}}{P_{a, \max }}=1-4 \frac{R_{f} R_{d}}{\left(R_{f}+R_{d}\right)^{2}+\left(Y_{f}+Y_{d}\right)^{2}},
$$

from which it is clear that $\gamma_{r}=0$, or full absorption is achieved, for $R_{f}=R_{d}$ and $Y_{f}=-Y_{d}$; defining the two conditions for a complex conjugate impedance.

Now, consider the equation of motion (11): the paddle inertia and gravity coefficients are fixed constants for a given wave board geometry, whereas added inertia and hydrodynamic damping are frequency dependent and have to be obtained from the generated wave field. Evaluating the equation for the first-order torque on a flap type machine (7) yields

$$
T_{\Phi}^{(1)}=\frac{1}{2}\left\{\rho g X_{a} \sum_{j=0}^{\infty} \frac{c_{j}}{k_{j}^{2}} \Gamma_{1}\left(k_{j}\right) e^{i \omega t}+c . c .\right\},
$$

where $\Gamma_{1}\left(k_{j}\right)$ is introduced for brevity and given by

$$
\Gamma_{1}\left(k_{j}\right)=\frac{k_{j}(h-d) \sinh k_{j} h-\cosh k_{j} h+\cosh k_{j} d}{\cosh k_{j} h} .
$$

The system's dynamic impedance may now be expressed as the ratio of wave board torque over the wave board angular velocity. Using an approximation for small angles

$$
X(t)=(h-d) \tan \theta(t) \approx(h-d) \theta(t),
$$

and introducing the frequency domain relationship between displacement $(X)$ and velocity $(V)$

$$
V(t)=\dot{X}(t) \stackrel{\mathscr{F}}{\rightarrow} V(\omega)=i \omega X(\omega)=i \omega(h-d) \theta(\omega)=(h-d) \Omega(\omega),
$$


where $\mathscr{F}$ denotes a Fourier transform, the first-order torque in the frequency domain, $T_{\Phi}^{(1)}(\omega)$, may be expressed as

$$
T_{\Phi}^{(1)}(\omega)=\rho g \frac{(h-d) \Omega(\omega)}{i \omega} \sum_{j=0}^{\infty} \frac{c_{j}}{k_{j}^{2}} \Gamma_{1}\left(k_{j}\right)
$$

where $\Omega$ is the wave board's angular velocity. Rewriting the equation of motion (11) in the frequency domain gives

$$
T(\omega)=\left[i\left(\omega\left(r+r_{\Phi}(\omega)\right)+\frac{m g h_{m}}{\omega}\right)+d_{\Phi}(\omega)\right] \Omega(\omega)=Z_{d}(\omega) \Omega(\omega),
$$

where $Z_{d}(\omega)$ is the complex impedance relating flap torque and the paddle angular velocity. Comparing (20) and (21) yields the equations for hydrodynamic damping

$$
d_{\Phi}(\omega)=\frac{\rho g(h-d)}{\omega} \frac{c_{0}}{k_{0}^{2}} \Gamma_{1}\left(k_{0}\right)
$$

and added inertia

$$
r_{\Phi}(\omega)=\frac{\rho g(h-d)}{\omega^{2}} \sum_{j=1}^{\infty} \frac{c_{j}}{k_{j}^{2}} \Gamma_{1}\left(k_{j}\right) .
$$

Examining (21) further, shows that some of the components of torque are in phase with the velocity (real part), whilst others are 90 degree out of phase (imaginary part). The optimum absorption filter will have the same real part, while the imaginary part will be of equal magnitude and opposite sign (complex conjugate filter). This is equivalent to Falnes (1980) finding that the optimum oscillator velocity is in phase with the excitation force. Designing a perfect complex conjugate controller is impossible, as this would require complete knowledge about future signals. Chatry et al. (1998) present several strategies for designing a non-ideal causal approximation for this non-causal problem. The method chosen here, relies on a best fit of the real and imaginary parts over a frequency band of interest. Figure 4 (a) shows the real and imaginary parts of the dynamic impedance $Z_{d}(\omega)$ compared to the real and imaginary parts of the absorption filter impedance $Z_{f}(\omega)$; both terms being evaluated for an existing bottom hinged wave maker. Figure 4 (a) also shows the two conditions for optimum absorption, $\Re\left\{Z_{d}(\omega)\right\}-\Re\left\{Z_{f}(\omega)\right\}=0$ and $\Im\left\{Z_{d}(\omega)\right\}+\Im\left\{Z_{f}(\omega)\right\}=0$. Towards the higher frequencies, the imaginary part of the dynamic impedance, $\Im\left\{Z_{d}(\omega)\right\}$, increases rapidly. This is mainly due to the increasing contribution of the evanescent modes in this regime, evidence of which is provided on figure 2. In this case it becomes difficult to provide sufficient negative filter reactance, resulting in a non-zero sum of the two imaginary parts. ${ }^{1}$ Figure 4 (b) shows the resulting power reflection coefficient $\gamma_{r}$. The absorption becomes poor when the filter fails to match the machines reactance. Nonetheless, very good power absorption is achieved over a wide band of interest.

\subsection{Frequency domain model}

All system components have now been discussed. Figure 5 shows a block diagram of the entire wave machine, the four contributions being: $C(\omega)$ - the machine controller, $G(\omega)$ - the wave board and water transfer function, $Z_{f}(\omega)$ - the absorption filter and $Z_{d}(\omega)$ - the dynamic torque feedback.

Any real wave machine needs a controller to minimize the error between demand and measured feedback, a process commonly referred to as closing the loop. Even though the controller has to be included in the simulation process, it will not be discussed here. An ideal closed-loop discrete-time controller of a hinged wave maker is discussed in Hodge \& Cherchas (1988). However, their approach is only partially applicable here since they adopt the paddle position as the control quantity. The transfer function $G(\omega)$ relates the drive motor torque to flap angular velocity and is the inverse of $Z_{d}(\omega)$.

Applying standard frequency domain methods, the block diagram in figure 5 may be expressed as a single transfer function

$$
B(\omega)=\frac{1}{i \omega} \frac{C(\omega) G(\omega)}{1+C(\omega) G(\omega)\left[Z_{f}(\omega)+Z_{d}(\omega)\right]},
$$

\footnotetext{
${ }^{1} \mathrm{An}$ increased imaginary part in the filter impedance results in an additional phase shift in the processed velocity signal. From basic control theory it is well known that sufficient phase margin (63 degree for optimum step response, but at least 30 degree for a reasonable overshoot characteristic) must be maintained to ensure system stability. Therefore, absorption has to be traded off against stability.
} 

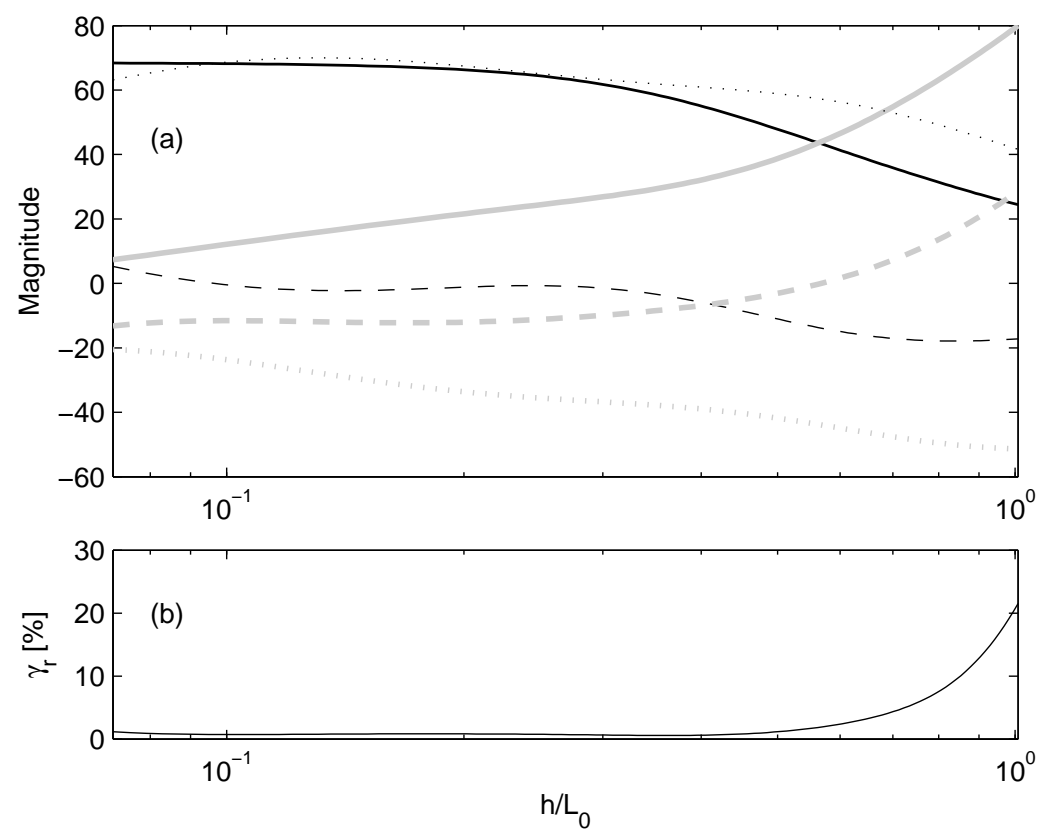

Figure 4: Impedance matching for a bottom hinged flap-type wave maker, $-\Re\left\{Z_{d}(\omega)\right\} ;-\Im\left\{Z_{d}(\omega)\right\}$; $\cdots \Re\left\{Z_{f}(\omega)\right\} ; \cdots \Im\left\{Z_{f}(\omega)\right\} ;--\Re\left\{Z_{d}(\omega)\right\}-\Re\left\{Z_{f}(\omega)\right\} ;--\Im\left\{Z_{d}(\omega)\right\}+\Im\left\{Z_{f}(\omega)\right\}$

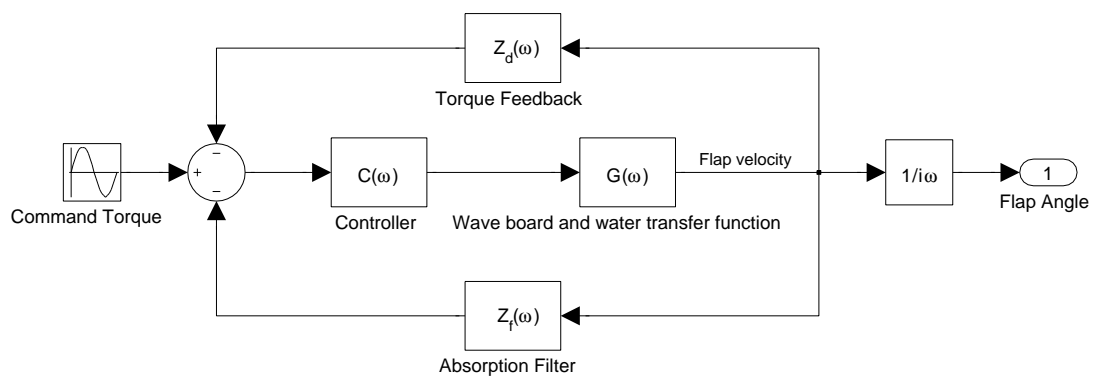

Figure 5: General system model

where $B(\omega)$ relates the command torque to the flap angle for a closed loop control system and can now be used to model and simulate the behaviour of the wave machine.

\subsection{Wave generation at second harmonic and second-oder correction}

Equation (10) defines the second-order torque $T^{(2)}$. This is the forcing term for a wave board motion at second harmonic $X_{1}^{(2)}$. Using the system's transfer function $B(\omega)$ this motion can be related directly to the second-order torque,

$$
X_{1, a}^{(2)}=(h-d) B\left(2 \omega_{x}\right) T_{a}^{(2)},
$$

where $B\left(2 \omega_{x}\right)$ is the frequency response of the transfer function $B(\omega)$ evaluated at frequency $2 \omega_{x} ; \omega_{x}$ being the frequency of the primary progressive wave. The additional subscript $a$ indicates that the complex amplitudes of the respective terms are considered. For any given second-order paddle displacement, the time history and the complex amplitude are related by

$$
X^{(2)}=\frac{1}{2}\left\{-i X_{a}^{(2)} e^{i 2 \omega t}+c . c .\right\}
$$


where the factor $-i$ was kept for consistency with Schäffer (1996). Similarly, any given second-order torque time history is related to the complex amplitude by

$$
T^{(2)}=\frac{1}{2}\left\{-i T_{a}^{(2)} e^{i 2 \omega t}+\text { c.c. }\right\} .
$$

In order to eliminate the spurious free wave content, the second-order wave board motion is given by

$$
X_{0, a}^{(2)}=\frac{F^{+} c_{0}^{2}}{h} X_{a}^{2},
$$

where the transfer function $F^{+}$is found (Schäffer 1996) by ensuring that the progressive part of the spurious free wave and the progressive part of the additionally generated wave cancel out; the definition of $\mathrm{F}^{+}$is given in Appendix A. Introducing a second-order compensation torque with complex amplitude $T_{c, a}^{(2)}$, leading to an additional paddle motion $X_{2, a}^{(2)}$, the desired second-order paddle motion $X_{0, a}^{(2)}$ may now be expressed as the sum of $X_{1, a}^{(2)}$ and $X_{2, a}^{(2)}$

$$
X_{0, a}^{(2)}=X_{1, a}^{(2)}+X_{2, a}^{(2)}=(h-d) B\left(2 \omega_{x}\right)\left(T_{a}^{(2)}+T_{c, a}^{(2)}\right) .
$$

Comparing (28) and (29) yields

$$
T_{c, a}^{(2)}=\frac{F^{+} c_{0}^{2} X_{a}^{2}}{h(h-d) B\left(2 \omega_{x}\right)}-T_{a}^{(2)},
$$

which is the second-order compensation torque required to eliminate the spurious free wave. Equations (25) and (30) are the two main outcomes of this theory. Whilst the former enables us to understand the behaviour of existing first-order controlled force-feedback machines, the latter provides an expression for full second-order compensation. Both results are evaluated in the following section. The additional second-order potential, $\Phi^{(23)}$, is given by

$$
\Phi^{(23)}=\frac{1}{2}\left\{\frac{i g}{2 \omega} X_{a}^{(2)} \sum_{p=0}^{\infty} c_{p}^{(23)+} \frac{\cosh K_{p}^{+}(z+h)}{\cosh K_{p}^{+} h} e^{i\left(2 \omega t-K_{p}^{+} x\right)}+\text { c.c. }\right\}
$$

where $c_{p}^{(23)}$ is given in Appendix A and the second-order paddle displacement amplitude at still water level, $X_{a}^{(2)}$, is defined as

$$
X_{a}^{(2)}=\left\{\begin{array}{cl}
X_{1, a}^{(2)} & \text { for first-order force control } \\
X_{0, a}^{(2)} & \text { for second-order force control. }
\end{array}\right.
$$

\section{Model evaluation}

\subsection{Simulation Models}

Many control systems can be modelled and simulated using a transfer function approach in the frequency domain. Such a transfer function expresses the frequency dependent ratio of output to input, involving both magnitude and phase. The coefficients characterising a model are often constant, or frequency independent, and as a result it is easily possible to obtain step responses using control system toolboxes. However, in the present case the hydrodynamic coefficients characterising the second-order mass-spring-damper system are strongly frequency dependent. Consequently, a transfer function evaluated for a particular wave frequency is only valid at this frequency. Exciting the transfer function with either an impulse or a step function would lead to meaningless results, as both correspond to an infinite series of frequency components. When modelling the given system this had to be considered: each time- or frequency-domain simulation is limited to one particular point and the effect of the second-order components in the feedback path need to be evaluated in a separate simulation with adopted hydrodynamic coefficients.

Two different methods were used to simulate the behaviour of the machine: a time-domain simulation using Matlab Simulink and a frequency-domain simulation. The simple Simulink model (figure 5) requires 


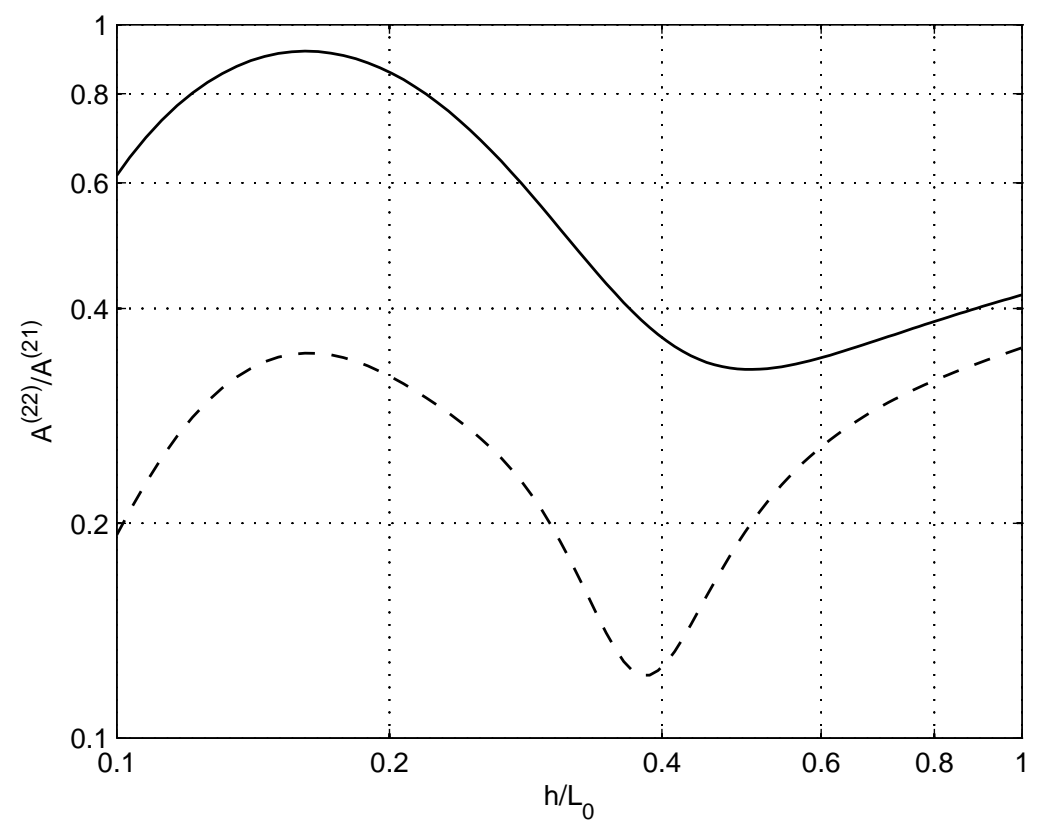

Figure 6: Dimensionless spurious wave content for flap-type machine hinged at $d / h=0,-$ Position-control mode; - - Force-control mode

differentiation in order to evaluate paddle acceleration from its angular velocity. An ideal differentiator has an infinite gain for very high frequencies. For any real differentiator, some form of gain limitation at higher frequencies must be included. Since this was found to affect the simulation results, especially at the second harmonic frequencies of the shorter wave components, the model had to be reformulated. Using this modified model, the Simulink time-domain simulation and the frequency-domain response model produced identical results.

\subsection{Second-order content for flap-type machines}

For flap-type machines, the wave board and water transfer function is given as

$$
G(\omega)=\frac{\Omega}{T}=\frac{1}{\left(r+r_{\Phi}\right) i \omega+d_{\Phi}-m g h_{m} /(i \omega)} .
$$

This is the ratio of angular velocity to input torque and follows directly from the equation of motion (11). Using $G(\omega)$ allows the evaluation of the system transfer function $B(\omega)$ for a matched absorption filter and closed-loop control.

The model evaluation has two main objectives. First, the model should provide an improved understanding of the operation for existing force-controlled flap-type machines, or machines driven with a first-order force control signal. Second, the new second-order force command signal should be verified. Whilst the latter may perhaps be more readily demonstrated via experimental observations (part II), the first point requires careful consideration.

Figure 6 provides a dimensionless plot of the spurious wave content for a bottom hinged wave maker operating in both position-control and force-control mode. The curve relating to position-control is identical to that shown in figure 3 , the only difference being that the frequency band is now limited to the region of interest appropriate to wave generation using a flap wave machine (figure 2 a). A detailed discussion concerning the spurious wave characteristic in position-control mode is given in $\$ 2.2 .3$. In force-control mode the total spurious wave is given by the sum of the original spurious wave and the wave generated by the feedbackinduced paddle motion at second harmonic, $X_{1}^{(2)}$. Figure 6 clearly shows that this latter component, the 


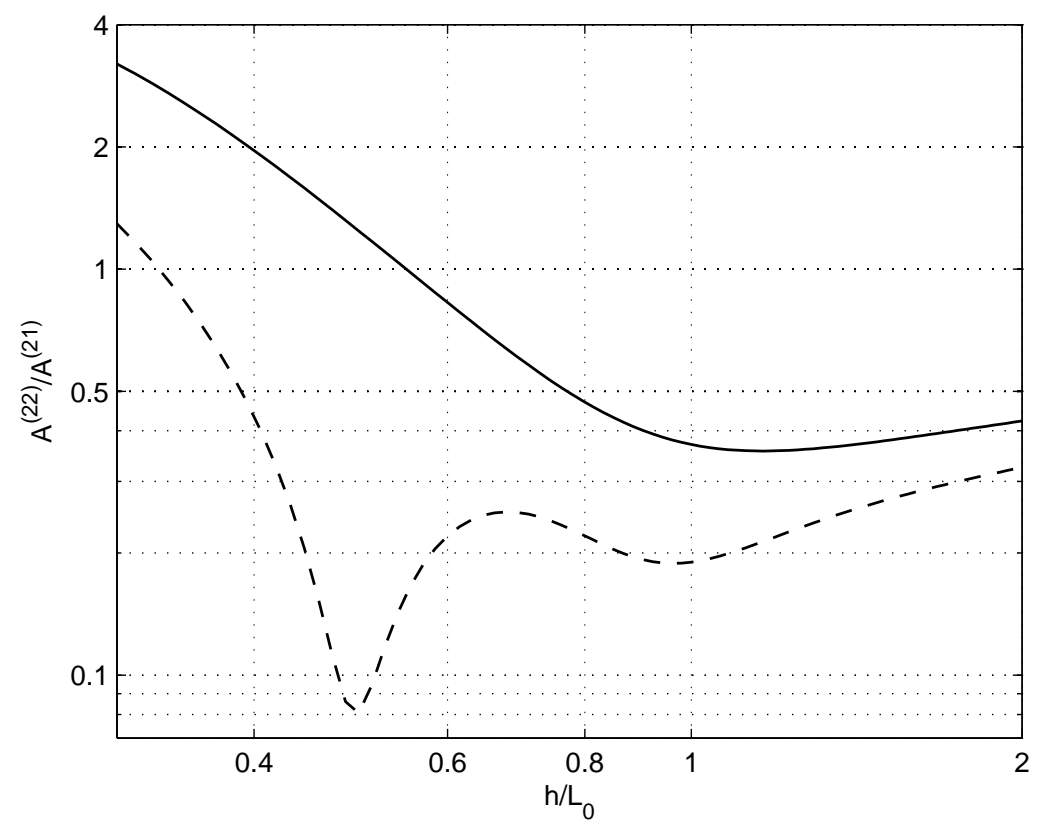

Figure 7: Dimensionless spurious wave content for flap-type machine hinged at $d / h=0.5,-$ Positioncontrol mode; - - Force-control mode

feedback-induced wave, cancels out a significant proportion of the original spurious wave. For example, the maximum value of the nondimensionless ratio, $A^{(22)} / A^{(21)}$, occurring at $h / L_{0} \approx 0.15$ is cut to about one third of its original value. Indeed for most of the region of interest, the spurious wave content is reduced considerably. It is only towards higher relative frequencies, corresponding to deeper water, that the difference between the two approaches reduces. In practice, this effect is hardly noticeable as second-order effects, notably the Stokes second-order contribution to the wave profile, $\eta^{(21)}$, are very weak in this regime.

Figure 7 shows the same dimensionless ratio, $A^{(22)} / A^{(21)}$, for a wave machine hinged half way between the still water level and the bed. This is an important case because a bottom hinged wave maker becomes impractical in deep water test tanks. Once again, the frequency band has been limited to the region of practical wave generation (figure $2 \mathrm{~b}$ ). As was the case for the bottom-hinged wave maker, a considerable cut in spurious wave content is observed across a broad range of frequencies.

In the present study, the optimum absorption filters were estimated using a best fit technique as discussed in $\S 2.4$ and shown in figure 4; the curves given in figures 6 and 7 being evaluated using the estimated filter impedance, $Z_{f}$. This force control strategy is identical to that implemented on most operating force-controlled wave machines. As a result, the dashed lines given on figures 6 and 7 will be representative of the performance of these machines, the detail being dependent upon the specific geometry of the machine involved.

When simulating the second-order content produced by a force-driven wave machine, values for paddle inertia and the paddle's gravity induced torque component $\left(m g h_{m}\right)$ are required. In the present study these values are based upon estimates for two existing wave machines. For other configurations, these values will vary. However, as long as the filter impedance provides a good match to the dynamic impedance, further simulations have shown that the characteristic of the spurious wave content only varies marginally. Nonetheless, a detailed analysis of the wave board's dynamic properties may be required for each practical application.

Similar arguments apply to the second-order correction torque shown in figure 8; the absolute value of this torque varying between different wave machines. Nevertheless, the characteristic shown on figure 8 gives a comprehensive insight in the operation of force-controlled machines. Within this figure, the second-order correction torque amplitude, $T_{c, a}^{(2)}$, is plotted normalised over the second-order wave-induced torque amplitude, $T_{\Phi, a}^{(2)}$, producing a graph that is steepness independent. However, it is important to note that $T_{\Phi}^{(2)}$ is not the only second-order torque acting on the machine; indeed, the feedback torque arising due to the wave 


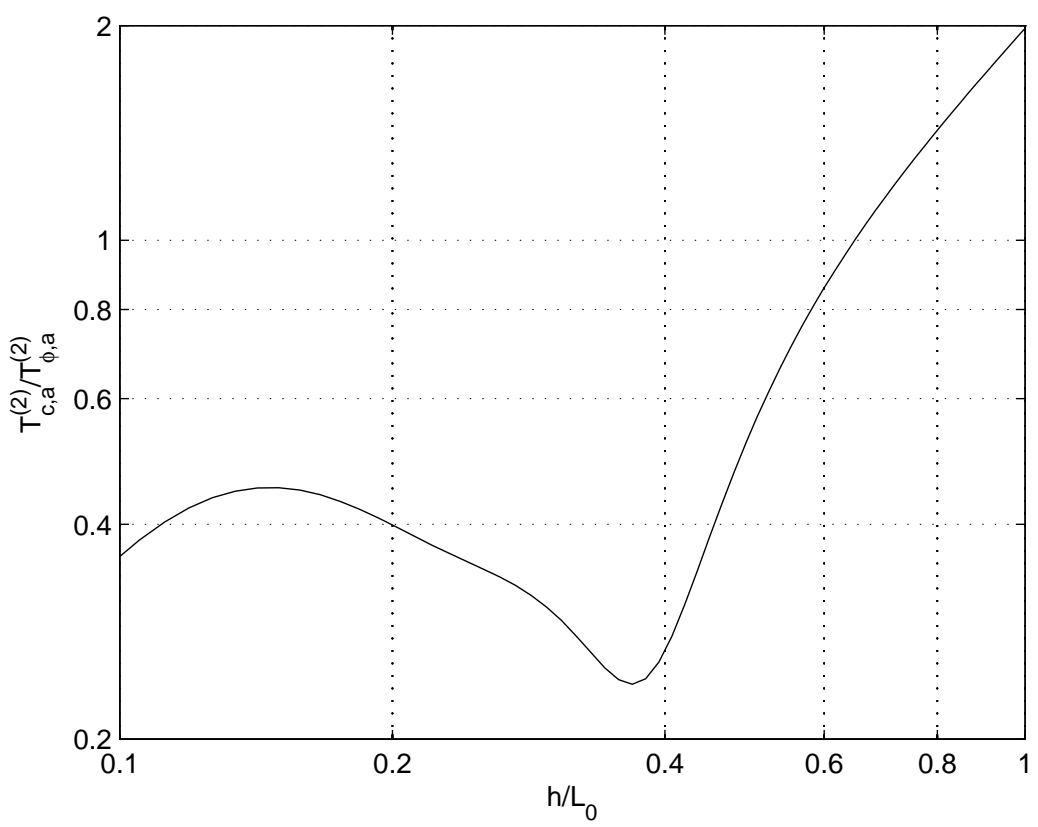

Figure 8: Dimensionless second-order correction torque for flap-type machine hinged at $d / h=0$

machine's inertia may become dominant at higher frequencies. Nevertheless, the minimum value of this ratio, $T_{c, a}^{(2)} / T_{\Phi, a}^{(2)}$, occurs at $h / L_{0} \approx 0.37$. This is closely related to the second-order spurious wave minimum noted in figure 6 . Given the magnitude of the terms presented on figure 8 , it is clear that in the proximity of this minimum a second-order correction is not required. Indeed, for a relatively large frequency bandwidth, the second-order correction torque is small, indicating that first-order force-control may be appropriate for the operation of flap wave machines.

\section{Conclusions}

A second-order force-feedback wave making theory has been established. Application of the theory has demonstrated that over the frequency range where the absorption mechanism is effective a first-order forcecontrolled flap-type wave machine gives much reduced spurious second-harmonic waves when compared to a position-controlled machine. Indeed, over a substantial frequency bandwidth the spurious second-order wave is dramatically reduced and a second-order correction may not be required. The theory outlined is limited to flap-type wave machines, generating waves of a single frequency. Further work will involve the experimental validation of the results outlined in section 2 (part II) and, at a later date, the expansion of the model to incorporate random waves, particularly wave groups, and a larger range of wave paddle types. 


\section{References}

Chatry, G., Clement, A. H. \& Gouraud, T. (1998), Self-adaptive control of a piston wave absorber, in 'Proceedings of the Eighth International Offshore and Polar Engineering Conference', Vol. 1, pp. 127133.

Dean, R. \& Dalrymple, R. (1991), Water Wave Mechanics for Engineers and Scientists, World Scientific.

Falnes, J. (1980), 'Radiation impedances matrix and optimum power absorption for interacting oscillators in surface waves', Applied Ocean Research 2(2), 75-80.

Falnes, J. (1993), Optimum control of oscillation of wave-energy converters, Wave energy converters: Generic technical evaluation study, DG XII Joule Wave Energy Initiative.

Falnes, J. (2002), Ocean Waves and Oscillating Systems, Cambridge University Press.

Flick, R. E. \& Guza, R. T. (1980), 'Paddle generated waves in laboratory channels', Journal of Waterway, Port, Coastal and Ocean Engineering 106(WW1), 79-97.

Havelock, T. H. (1929), 'Forced surface-wave on water', Philosophical Magazine viii, 569-576.

Hodge, S. \& Cherchas, D. (1988), 'Close-loop discrete-time control of a hinged wavemaker', Automatica $\mathbf{2 4}(3), 397-404$.

Lighthill, J. (1979), 'Waves and hydrodynamic loading.', Acta Informatica 1, 1 - 40.

Masterton, S. \& Swan, C. (2008), 'On the accurate and efficient calibration of a 3d wave basin', Ocean Engineering 35(8-9), $763-773$.

McCormick, M. (1983), 'Analysis of optimal ocean wave energy conversion', Journal of Waterway, Port, Coastal and Ocean Engineering 109, 180-198.

McCormick, M., Johnson, D., Hebron, R. \& Hoyt, J. (1981), Wave energy conversion in restricted waters by a heaving cylinder/linear-inductance system, in 'Proceedings OCEANS 81 (IEEE/MTS), Boston, Massachusetts'.

Milgram, J. H. (1970), 'Active water-wave absorbers', Journal of Fluid Mechanics 43, 845-859.

Naito, S. (2006), 'Wave generation and absorption in wave basins: Theory and applications', International Journal of Offshore and Polar Engineering 16(2), 81-89.

Ottesen-Hansen, N. E., Sand, S. E., Lundgren, H., Sorensen, T. \& Gravesen, H. (1980), Correct reproduction of long group induced waves, in 'Proceedings of the 17th Coastal Engineering Conference, Sydney, Australia', pp. 784-800.

Salter, S. (1982), Absorbing wave-makers and wide tanks, in 'Directional Wave Spectra Applications', pp. $185-200$.

Salter, S. H., Jeffery, D. C. \& Taylor, J. R. M. (1976), 'The architecture of nodding duck wave power generators', The Naval Architect Jan 1976, 21-24.

Sand, S. E. (1982), 'Long wave problems in laboratory models', Journal of Waterways, Port, Coastal and Ocean Engineering 108(WW4), 492-503.

Sand, S. E. \& Donslund, B. (1985), 'Influence of the wave board type on bounded long waves', Journal of Hydraulic Research 23, 147-163.

Schäffer, H. A. (1996), 'Second-order wavemaker theory for irregular waves', Ocean Engineering 23, 47-88.

Schäffer, H. A. \& Jakobsen, K. (2003), Non-linear wave generation and active absorption in wave flumes, in 'Long Waves Symposium', pp. 69-77. 
Schäffer, H. A. \& Klopman, G. (2000), 'Review of multidirectional active wave absorption methods', Journal of Waterway, Port, Coastal and Ocean Engineering 126(2), 88 - 97.

Spinneken, J. \& Swan, C. (2008b), 'Second-order wave maker theory using force-feedback control. Part II. An experimental verification of regular wave generation', Ocean Engineering; Submitted for revision .

Sulisz, W. \& Hudspeth, R. T. (1993), 'Complete second-order solution for water waves generated in wave flumes', Journal of Fluids and Structures 7, 253-268.

van Leeuwen, P. J. \& Klopman, G. (1996), 'New method for the generation of second-order random waves', Ocean Engineering (Pergamon) 23(2), 167 - 192.

Zaman, M. \& Mak, L. (2007), Second order wave generation technique in the laboratory, in 'Proceedings of the 26th International Conference on Offshore Mechanics and Arctic Engineering'.

Zhang, H. (2005), A Deterministic Combination of Numerical and Physical Models for Coastal Waves, PhD thesis, Technical University of Denmark.

\section{Appendix A - The position-control wave field coefficients}

At first-order, the wave field is described by the velocity potential $\Phi^{(1)}$, equation 1 . Herein, the coefficients $c_{j}$ need to be defined; they follow directly from the formulation in Schäffer (1996) and are given by

$$
c_{j}=\sinh k_{j} h \frac{\Lambda_{1}\left(k_{j}\right)}{\Lambda_{2}\left(k_{j}\right)}
$$

where

$$
\Lambda_{1}\left(k_{j}\right)=\sinh k_{j} h-\frac{d+l}{h+l} \sinh k_{j} d+\frac{1}{h+l} \frac{\cosh k_{j} d-\cosh k_{j} h}{k_{j}}
$$

and

$$
\Lambda_{2}\left(k_{j}\right)=\frac{1}{2}\left(k_{j} h+\sinh k_{j} h \cosh k_{j} h\right) .
$$

With reference to equations (4) and (5), describing the second-order wave field, the expressions for $H_{j l}^{+}, D_{j l}^{+}$, $K_{p}^{+}$and $c_{p}^{(22)+}$ need to be introduced. These follow directly from Schäffer (1996) but are adopted for the special case of regular waves and, consequently, the generation of superharmonics only.

$$
\begin{gathered}
H_{j l}^{+}=2 \omega\left(\omega^{2}-\frac{g^{2} k_{j} k_{l}}{\omega^{2}}\right)+\omega^{3}-\frac{g^{2}}{2}\left(\frac{k_{j}^{2}}{\omega}+\frac{k_{l}^{2}}{\omega}\right) \\
D_{j l}^{+}=g\left(k_{j}+k_{l}\right) \tanh \left(k_{j}+k_{l}\right) h-4 \omega^{2} \\
c_{p}^{(22)+}=\frac{h \omega \cosh ^{2} K_{p}^{+} h}{g^{2} c_{0}^{2} \Lambda_{2}\left(K_{p}^{+}\right)}\left\{\frac{g}{2 \omega} \sum_{j=0}^{\infty} \frac{c_{j} k_{j}^{2}}{k_{j}^{2}-\left(K_{p}^{+}\right)^{2}}\left(-3 \omega^{2}+M_{2}\left(k_{j}, K_{p}^{+}\right)\right)\right. \\
+\frac{g}{2 \omega} \sum_{l=0}^{\infty} \frac{c_{l} k_{l}^{2}}{k_{l}^{2}-\left(K_{p}^{+}\right)^{2}}\left(-3 \omega^{2}+M_{2}\left(k_{l}, K_{p}^{+}\right)\right) \\
\left.-\sum_{j=0}^{\infty} \sum_{l=0}^{\infty} c_{j} c_{l} \frac{k_{j}+k_{l}}{\left(k_{j}+k_{l}\right)^{2}-\left(K_{p}^{+}\right)^{2}} H_{j l}^{+}\right\}
\end{gathered}
$$


where

$$
\begin{aligned}
M_{2}\left(k_{j}, K_{p}^{+}\right)=-\frac{g}{h+l} \frac{K_{p}^{+} / k_{j}}{k_{j}^{2}-\left(K_{p}^{+}\right)^{2}}\left\{2 k_{j} K_{p}^{+}(1\right. & \left.-\frac{\cosh k_{j} d}{\cosh k_{j} h} \frac{\cosh K_{p}^{+} d}{\cosh K_{p}^{+} h}\right) \\
& \left.-\left(k_{j}^{2}+\left(K_{p}^{+}\right)^{2}\right)\left(\frac{4 \omega^{4}}{g^{2} k_{j} K_{p}^{+}}-\frac{\sinh k_{j} d}{\cosh k_{j} h} \frac{\sinh K_{p}^{+} d}{\cosh K_{p}^{+} h}\right)\right\}
\end{aligned}
$$

and $K_{p}^{+}$is the solution to

$$
(2 \omega)^{2}=g K_{p}^{+} \tanh K_{p}^{+} h
$$

which is the dispersion equation generalized to complex numbers. With reference to equations (28) and (30), the transfer function $F^{+}$found by Schäffer (1996) is introduced as

$$
F^{+}=-\frac{c_{0}^{(22)+}}{c_{0}^{(23)+}}
$$

where $c_{0}^{(23)+}$ is the progressive mode $(p=0)$ of

$$
c_{p}^{(23)+}=\sinh K_{p}^{+} h \frac{\Lambda_{1}\left(K_{p}^{+}\right)}{\Lambda_{2}\left(K_{p}^{+}\right)} .
$$

\section{Appendix B - The second-order torques}

With reference to equation (8), the torques up to second order need to be evaluated. The first-order unsteady wave acceleration $\partial \Phi^{(1)} / \partial t$ integrated up to the first-order free surface induces a torque given by

$$
T_{\Phi^{(1)}}^{(2)}=-\int_{0}^{\eta^{(1)}} \rho \frac{\partial \Phi^{(1)}}{\partial t}(h-d+z) d z=\frac{1}{2}\left\{\rho g X_{a} \sum_{j=0}^{\infty} c_{j}\left[(h-d) \eta_{1}+\eta_{1}^{2}\right] e^{i \omega t}+\text { c.c. }\right\}
$$

which can be simplified by retaining only mean- and second-order terms

$$
T_{\Phi^{(1)}}^{(2)}=\frac{1}{2}\left\{\frac{\rho g(h-d) X_{a}^{2}}{2} \sum_{j=0}^{\infty} \sum_{l=0}^{\infty} c_{j} c_{l} e^{i 2 \omega t}+\frac{\rho g(h-d) X_{a} X_{a}^{*}}{2} \sum_{j=0}^{\infty} \sum_{l=0}^{\infty} c_{j} c_{l}^{*}+c . c .\right\} .
$$

Following Lighthill (1979), there is also a torque component caused by the changing effective wetted surface of the body which may be expressed as

$$
T_{\text {hydro }}^{(2)}=-\int_{0}^{\eta^{(1)}} \rho g z(h-d+z) d z=-\frac{1}{2} \rho g \eta_{1}^{2}(h-d)-\frac{1}{3} \rho g \eta_{1}^{3}
$$

and simplified by retaining only mean- and second-order terms

$$
T_{\text {hydro }}^{(2)}=\frac{1}{2}\left\{-\frac{\rho g(h-d) X_{a}^{2}}{4} \sum_{j=0}^{\infty} \sum_{l=0}^{\infty} c_{j} c_{l} e^{i 2 \omega t}-\frac{\rho g(h-d) X_{a} X_{a}^{*}}{4} \sum_{j=0}^{\infty} \sum_{l=0}^{\infty} c_{j} c_{l}^{*}+\text { c.c. }\right\} .
$$

Examining (B-2) and (B-4) one can note that the hydrostatic second-order torque is of half the magnitude and opposite sign when compared to the torque induced by the first-order unsteady wave potential, $\partial \Phi^{(1)} / \partial t$. This is consistent with the findings presented by Lighthill (1979). The last set of second-order torques induced by the linear potential, $\Phi^{(1)}$, are the components associated with the horizontal and vertical velocity squared terms, $u^{2}$ and $w^{2}$ respectively. With the horizontal velocity being defined as $u=\partial \Phi^{(1)} / \partial x$, the former of these two contributions may be expressed as

$$
T_{u^{2}}^{(2)}=-\int_{-h+d}^{0} \frac{\rho}{2}\left(\frac{\partial \Phi^{(1)}}{\partial x}\right)^{2}(h-d+z) d z
$$


which evaluates to

$$
\begin{aligned}
T_{u^{2}}^{(2)}=\frac{1}{2}\left\{\frac{\rho g^{2} X_{a}^{2}}{4 \omega^{2}} \sum_{j=0}^{\infty} \sum_{l=0}^{\infty} \frac{c_{j} k_{j} c_{l} k_{l}}{\cosh k_{j} h \cosh k_{l} h} \Gamma_{u^{2}} e^{i 2 \omega t}\right. & \\
& \left.+\frac{\rho g^{2} X_{a} X_{a}^{*}}{4 \omega^{2}} \sum_{j=0}^{\infty} \sum_{l=0}^{\infty} \frac{c_{j} k_{j} c_{l}^{*} k_{l}^{*}}{\cosh k_{j} h \cosh k_{l}^{*} h} \Gamma_{u^{2}}+\text { c.c. }\right\}
\end{aligned}
$$

where $\Gamma_{u^{2}}$ is given by

$$
\Gamma_{u^{2}}=\left\{\begin{array}{cc}
\frac{1}{8 k_{j}^{2}}\left\{2 k_{j}(d-h) \sinh 2 k_{j} h+\cosh 2 k_{j} h-\cosh 2 k_{j} d-2 k_{j}^{2}(d-h)^{2}\right\} & \text { for } j=l \\
\frac{1}{2\left(k_{j}^{2}-k_{l}^{2}\right)}\left\{2(d-h)\left[k_{j} \sinh k_{j} h \cosh k_{l} h-k_{l} \sinh k_{l} h \cosh k_{j} h\right]\right. & \\
+\frac{k_{j}-k_{l}}{k_{j}+k_{l}}\left[\cosh \left(k_{j}+k_{l}\right) h-\cosh \left(k_{j}+k_{l}\right) d\right] & \text { for } j \neq l \\
\left.+\frac{k_{j}+k_{l}}{k_{j}-k_{l}}\left[\cosh \left(k_{j}-k_{l}\right) h-\cosh \left(k_{j}-k_{l}\right) d\right]\right\} &
\end{array}\right.
$$

Similarly, the torque induced by the vertical velocity term, $w=\partial \Phi^{(1)} / \partial z$ is given by

$$
T_{w^{2}}^{(2)}=-\int_{-h+d}^{0} \frac{\rho}{2}\left(\frac{\partial \Phi^{(1)}}{\partial z}\right)^{2}(h-d+z) d z
$$

and evaluates to

$$
\begin{aligned}
T_{w^{2}}^{(2)}=\frac{1}{2}\left\{-\frac{\rho g^{2} X_{a}^{2}}{4 \omega^{2}} \sum_{j=0}^{\infty} \sum_{l=0}^{\infty} \frac{c_{j} k_{j} c_{l} k_{l}}{\cosh k_{j} h \cosh k_{l} h} \Gamma_{w^{2}} e^{i 2 \omega t}\right. & \\
& \left.+\frac{\rho g^{2} X_{a} X_{a}^{*}}{4 \omega^{2}} \sum_{j=0}^{\infty} \sum_{l=0}^{\infty} \frac{c_{j} k_{j} c_{l}^{*} k_{l}^{*}}{\cosh k_{j} h \cosh k_{l}^{*} h} \Gamma_{w^{2}}+\text { c.c. }\right\}
\end{aligned}
$$

where $\Gamma_{w^{2}}$ is given by

$$
\Gamma_{w^{2}}= \begin{cases}\frac{1}{8 k_{j}^{2}}\left\{2 k_{j}(d-h) \sinh 2 k_{j} h+\cosh 2 k_{j} h-\cosh 2 k_{j} d+2 k_{j}^{2}(d-h)^{2}\right\} & \text { for } j=l \\ \frac{d-h}{2}\left[\frac{1}{k_{j}+k_{l}} \sinh \left(k_{j}+k_{l}\right) h-\frac{1}{k_{j}-k_{l}} \sinh \left(k_{j}-k_{l}\right) h\right] & \\ +\frac{1}{2\left(k_{j}+k_{l}\right)^{2}}\left[\cosh \left(k_{j}+k_{l}\right) h-\cosh \left(k_{j}+k_{l}\right) d\right] & \text { for } j \neq l \\ -\frac{1}{2\left(k_{j}-k_{l}\right)^{2}}\left[\cosh \left(k_{j}-k_{l}\right) h-\cosh \left(k_{j}-k_{l}\right) d\right] & \end{cases}
$$

The torque induced by the second-order Stokes wave potential, $\Phi^{(21)}$, follows from the integration of the respective pressure field and is given by

$$
T_{\Phi^{(21)}}^{(2)}=-\int_{-h+d}^{0} \rho \frac{\partial \Phi^{(21)}}{\partial t}(h-d+z) d z
$$

which evaluates to

$$
T_{\Phi(21)}^{(2)} \quad=\quad \frac{1}{2}\left\{\rho \omega X_{a}^{2} \sum_{j=0}^{\infty} \sum_{l=0}^{\infty} \frac{H_{j l}^{+}}{D_{j l}^{+}} \frac{c_{j} c_{l}}{\left(k_{j}+k_{l}\right)^{2}} \Gamma_{1}\left(k_{j}+k_{l}\right) e^{i 2 \omega t}+\text { c.c. }\right\} .
$$


Similarly, the torque induced by the spurious free wave potential, $\Phi^{(22)}$, is given by

$$
T_{\Phi^{(22)}}^{(2)}=-\int_{-h+d}^{0} \rho \frac{\partial \Phi^{(22)}}{\partial t}(h-d+z) d z
$$

and evaluates to

$$
T_{\Phi^{(22)}}^{(2)}=\frac{1}{2}\left\{\frac{\rho g c_{0}^{2} X_{a}^{2}}{h} \sum_{p=0}^{\infty} \frac{c_{p}^{(22)+}}{\left(K_{p}^{+}\right)^{2}} \Gamma_{1}\left(K_{p}^{+}\right) e^{i 2 \omega t}+c . c .\right\} .
$$

